\title{
ANALYSE ET RECOMMANDATIONS
}

\section{Introduction}

Aujourd'hui, la croissance de la consommation énergétique mondiale suscite une profonde inquiétude pour l'avenir. $\mathrm{D}$ 'une part, la pénurie de certaines ressources, comme le gaz naturel et le pétrole, fait craindre une envolée des coûts, constituant un frein dangereux au développement économique et une dépendance excessive de l'Europe vis-à-vis de son approvisionnement en énergie. D'autre part, les climatologues pensent que les activités anthropiques et plus particulièrement les émissions de gaz carbonique pourraient bouleverser le climat trop rapidement pour que l'humanité puisse s'y adapter dans de bonnes conditions. Pour faire face à ces menaces et tenter d'en atténuer l'ampleur, la recherche de nouvelles formes d'énergie exploitable a déjà commencé, avec un intérêt particulier pour celles qui n'augmentent pas la concentration des gaz à effet de serre dans l'atmosphère. Dans le bouquet de solutions dont la mise en œuvre sera nécessaire pour maintenir un niveau satisfaisant de développement sur la planète, il apparaît de plus en plus vraisemblable que l'énergie nucléaire jovera un rôle important. Cette ressource possède, en effet, des atouts décisifs qui répondent aux préoccupations actuelles puisqu'elle n'entraîne aucune émission de gaz à effet de serre et qu'elle utilise des combustibles présents en quantités presque illimitées.

L'énergie nucléaire de fission contribue déjà massivement à la production d'électricité, en France et cette part dominante perdurera sur plusieurs décennies. Toutefois, sous sa forme actuelle, elle ne peut se généraliser aisément au monde entier. En effet, la fission du seul isotope 235 de l'uranium naturel, utilisant une trop faible fraction du combustible nucléaire, conduirait, là aussi, à une crise d'approvisionnement et à une gestion difficile des déchets radioactifs 
générés. L'avenir de la fission est donc subordonné à l'avènement des réacteurs à neutrons rapides de la prochaine génération, la quatrième. Ils permettront de mieux utiliser la ressource en matière fissile, de produire moins de déchets nucléaires et d'incinérer les déchets issus des réacteurs à eau pressurisée en service aujourd'hui. Dans le cadre d'une collaboration internationale étendue, un vaste programme vise à élaborer des solutions industrielles convaincantes, respectant de sévères contraintes de sûreté environnementale, qui pourraient devenir opérationnelles avant un demi-siècle.

L'énergie de fusion se présente sous un jour plus contrasté que la fission. Elle $n^{\prime}$ a pas encore atteint le stade industriel et elle $n^{\prime}$ a pas encore donné de preuves indiscutables de son aptitude à y parvenir. Elle repose sur la maîtrise d'une physique spécifique qui a peu de rapports avec la science mise en jeu dans la fission comme dans toute autre méthode de production énergétique. Elle pose des questions nouvelles aux métallurgistes afin qu'ils élaborent des matériaux capables de supporter les rayonnements neutroniques qu'elle génère. Mais, au moins en théorie, les ressources en combustibles sont facilement accessibles et inépuisables, les déchets radioactifs ne posent pas de problèmes environnementaux insupportables et la sûreté passive est inhérente au principe du fonctionnement. L'ensemble de ces qualités prometteuses représente un enjeu si imposant qu'il faut se défendre contre un enthousiasme aveugle et vérifier patiemment la validité de ces assertions en s'engageant dans l'étude de la fusion avec tous les moyens disponibles dès maintenant, au niveau français et international, mais, pour que la fusion contribue massivement un jour à la production d'électricité nucléaire, le chemin reste long et escarpé.

Sur le plan international, les recherches sur l'énergie de fusion se structurent aujourd'hui autour de trois grands projets expérimentaux. Il s'agit, d'une part, de la machine $\mid l t e r^{r}$, fruit d'une coopération internationale, qui sera implantée à Cadarache, et, d'autre part, du Laser Mégajoule (LMJ) et du National Ignition Facility (NIF) qui sont en cours de construction respectivement à Bordeaux et à Livermore (États-Unis). Ces deux dernières installations sont financées sur des budgets de défense avec pour objectif principal la simulation des armes nucléaires. Prenant acte des décisions prises ${ }^{2}$ concernant ces machines, le présent rapport rappellera les objectifs des recherches sur la fusion nucléaire, puis il fera le point sur leur état aujourd'hui et décrira leurs évolutions probables au cours des vingt prochaines années, période dominée vraisemblablement par lter et le LMJ en Europe. Il analysera ensuite les principaux points durs à surmonter pour que ces deux expériences parviennent à une démonstration satisfaisante

\footnotetext{
${ }^{1}$ Iter, « le chemin » en latin, était à l'origine l'acronyme de International Thermonuclear Experimental Reactor.

${ }^{2}$ Pour Iter, communiqué de la Présidence de la République concernant le choix du site de Cadarache pour accueillir Iter en date du 28 juin 2005; pour le LMJ, annonce du Premier ministre concernant le lancement de la réalisation du Laser Mégajoule en date du 21 avril 1995.
} 
de faisabilité scientifique. Le rapport évaluera ensuite les difficultés qui accompagneront la mise en œuvre industrielle et les précautions nécessaires pour faciliter son acceptation par la société. II décrira brièvement les autres filières ou variantes pouvant offrir d'autres solutions si lter ou le LMJ en révélait la nécessité. Enfin, il s'étendra plus longuement sur l'organisation de l'enseignement et de la recherche dans le domaine de la fusion nucléaire, en proposant des aménagements et des coordinations qui paraissent indispensables pour faire face aux besoins des deux expériences et permettre à la communauté scientifique française $d^{\prime}$ en tirer le meilleur profit. Les propositions seront résumées à la fin du rapport.

Des chapitres supplémentaires donneront des informations sur les points les plus importants discutés dans l'analyse et les recommandations. Les deux premiers chapitres présenteront rapidement les deux voies principales envisagées pour la fusion : le confinement magnétique avec lter et le confinement inertiel avec le LMJ. Ensuite, les domaines scientifiques et techniques les plus directement concernés feront l'objet d'une présentation détaillée. Il s'agit, d'abord, de la physique des plasmas chauds magnétisés, qui reste le guide fondamental pour les recherches sur le confinement magnétique. La physique de l'interaction laserplasma suivra en raison de son rôle crucial dans les projets actuels de confinement inertiel, comme pour les développements futurs. Le rapport mettant l'accent sur les besoins urgents de développement en physique atomique, en simulation numérique et en science des matériaux, quatre autres chapitres en approfondiront la nature et les motivations. Puis, une mise au point détaillée présentera l'état actuel des réflexions sur les aspects liés à la sûreté et aux problèmes environnementaux ou sociétaux. Enfin, un court dernier chapitre évoquera certains aspects intéressants de ces recherches pour l'astrophysique.

Un rapport annexe, sous forme d'un CD-ROM, contient des présentations beaucoup plus complètes sur les résultats déjà acquis, les machines existantes et projetées, les problèmes industriels attendus, les retombées technologiques et les considérations économiques.

\section{Rappel des objectifs}

La Conférence de Genève sur les utilisations pacifiques de l'énergie atomique, en 1958, a véritablement marqué le début des recherches sur la fusion nucléaire contrôlée avec comme objectif la production d'énergie à des fins civiles. Après une suite de tâtonnements, de déceptions et de succès, l'avenir de cette source d'énergie apparaît plus clairement aujourd'hui. Les résultats acquis montrent qu'il est possible de construire des dispositifs produisant de l'énergie à partir 
de la fusion nucléaire et de dater approximativement les futures étapes qui devraient conduire à son exploitation industrielle. Les expériences actuelles, comme celles du proche avenir, appartiennent encore au domaine de la recherche. En Europe et au Japon, au moins, ces recherches s'affirment comme franchement orientées vers la réalisation d'un réacteur industriel. Cette intention se manifeste par la préoccupation constante d'éliminer les dispositifs qui n'ont aucune chance de déboucher un jour sur une production d'électricité dans des conditions industrielles, donc avec des coûts admissibles, en tenant compte des incertitudes attachées inévitablement à leur estimation.

La fusion des noyaux d'atomes préoccupe les physiciens nucléaires depuis que leur spécialité existe. En particulier, les expériences sur les éléments légers (hydrogène et ses isotopes, hélium, lithium) ont beaucoup contribué à clarifier la théorie des interactions nucléaires, en raison du faible nombre de nucléons intervenant dans les réactions. Très tôt également, les astrophysiciens ont trouvé dans les réactions de fusion nucléaire les clefs de l'évolution de la matière stellaire et la source d'énergie qui permet aux astres de rayonner. Puis, après avoir joué un rôle important dans le perfectionnement des armes nucléaires, la fusion a suivi la même route que la fission, avec beaucoup de retard, en s'affichant comme une source d'énergie présentant des avantages considérables. À ce jour, une seule réaction permet d'envisager une mise en œuvre à l'échelle industrielle. Elle résulte de la fusion des deux isotopes lourds de l'hydrogène, le deutérium et le tritium.

La fusion se produit lorsque la collision de deux noyaux d'atomes légers est suivie d'une réaction nucléaire avec formation d'un noyau plus lourd et diminution de la masse totale du système. La masse perdue se retrouve dans l'énergie cinétique des produits de la réaction, comme le prédit la relativité. La réaction la plus facile à réaliser est la fusion des noyaux de deutérium (D) et de tritium (T), les deux isotopes de l'hydrogène mentionnés plus haut. Le deutérium contient deux nucléons, le tritium trois. Au cours de la fusion, quatre de ces nucléons s'associent pour donner un noyau d'hélium 4, emportant une énergie cinétique de 3,5 MeV, et le nucléon restant est un neutron libre de $14 \mathrm{MeV}$ qui contient donc $80 \%$ de l'énergie produite par la réaction.

Cette réaction très exothermique présente l'intérêt supplémentaire de n'impliquer que des produits abondants et bon marché. En effet, le deutérium existe dans l'eau de mer à une concentration de $33 \mathrm{mg}$ par litre. Le tritium se fabrique à partir du lithium, élément bon marché et présent en grande quantité dans l'écorce terrestre. Ce métal alcalin, additionné d'un multiplicateur de neutrons, génère l'isotope de l'hydrogène lorsqu'il est irradié à l'aide des neutrons produits par la réaction de fusion elle-même. La fusion complète d'un gramme de mélange DT équimoléculaire libère $340 \mathrm{GJ}$, soit environ $94 \mathrm{MWh}$. Cette consommation extrêmement faible en combustible conduit à estimer que, au vu 
des ressources prévisibles, la domestication de la fusion libérerait l'humanité de tout risque de pénurie énergétique. C'est le lithium qui viendrait d'abord à manquer, dans plusieurs dizaines de siècles ${ }^{3}$. On peut espérer que les progrès de la science et de la technique permettraient alors de n'utiliser que le deutérium comme combustible, ce qui est possible mais plus difficile. Alors les réserves deviendraient vraiment illimitées.

Un autre intérêt de la fusion du DT réside dans le fait que cette réaction nucléaire ne génère aucun élément radioactif à vie longue. Les combustibles de base, lithium et deutérium, ne sont pas radioactifs. Le tritium, élément radioactif avec un temps de vie de 12 ans, serait fabriqué sur place au fur et à mesure des besoins sans en amasser des stocks importants. Seuls des éléments du réacteur deviendront radioactifs par exposition aux neutrons de $14 \mathrm{MeV}$, mais la radioactivité induite, dans les hypothèses actuelles, aura pratiquement disparu en une ou deux centaines d'années. La quantité de ces déchets peut être réduite en choisissant des matériaux dont l'activation reste faible. De plus, la production d'énergie ne s'accompagne d'aucune émission de gaz à effet de serre, point commun avec une centrale nucléaire classique, basée sur la fission des noyaux lourds.

Enfin, s'il reste indispensable de se prémunir contre les éventuels incidents, en particulier ceux concernant la gestion du tritium, la sûreté nucléaire d'une telle installation est facile à assurer. Aucune catastrophe majeure n'est à redouter, car un accident par emballement des réactions n'est pas possible. En effet, à chaque instant, le cœur du réacteur ne contient qu'une faible quantité de combustible et l'interruption de l'alimentation en mélange DT entraîne l'arrêt immédiat de la combustion, comme dans une chaudière à gaz. De plus, tout dérèglement ou détérioration aurait le même effet, tant est difficile le maintien des conditions physiques permettant aux réactions de se produire. Un arrêt du refroidissement avec maintien de l'alimentation provoquerait une montée des parois en température, suivi d'une vaporisation qui étoufferait ou déréglerait le mécanisme délicat qui maintient le milieu réactionnel dans les conditions favorables à une production d'énergie.

\section{2 | État de la situation aujourd'hui}

La fusion contrôlée fait l'objet de travaux intenses depuis la fin de la Seconde Guerre mondiale, avec un financement, au niveau européen, de plusieurs centaines de millions d'euros par an. Une exploitation industrielle est envisagée, au plus tôt, dans la seconde moitié du XXI ${ }^{\mathrm{e}}$ siècle. Ce long délai résulte du caractère

\footnotetext{
${ }^{3}$ Plusieurs millénaires avec les réserves connues et la consommation d'aujourd'hui.
} 
extrême des conditions physiques à réaliser. Pour s'en faire une idée, il suffit de noter que la fusion d'un noyau de deutérium (D) et d'un noyau de tritium (T) est la plus facile à mettre en œuvre et cependant elle nécessite de porter les combustibles à des températures supérieures à 100 millions de degré. Aujourd'hui deux méthodes sont envisagées pour domestiquer la fusion : le confinement magnétique et le confinement inertiel. Ni l'une, ni l'autre n'est encore parvenue à produire plus d'énergie qu'il n'en faut injecter pour maintenir les conditions des réactions. Iter appartient à la première voie et le LMJ à la seconde. Dans les deux cas, les deux dernières décennies ont permis des progrès suffisants pour envisager la réalisation de ces deux expériences, où des gains en énergie deviennent possibles.

La machine Iter, de la famille des Tokamaks ${ }^{4}$, sera le fruit de quarante ans de recherches sur le confinement magnétique. Dans ce type de machine (chapitre 1), on cherche à réaliser les conditions de la fusion, soit des températures de centaines de millions de degrés, en régime quasi permanent, dans une enceinte. La matière est alors complètement ionisée et forme un plasma. Un champ magnétique fort assure son isolation thermique des parois, en confinant les particules chargées dont il est constitué. En cinquante ans, les performances n'ont cessé de s'approcher du but, la température croissant de près d'un facteur mille sur cette durée et le plasma atteignant les caractéristiques requises pour le réacteur. La puissance de fusion la plus élevée a été obtenue sur la machine européenne Jet, avec un rapport puissance produite sur puissance injectée de $0,65^{5}$ et $16 \mathrm{MW}$ générés par les réactions de fusion pendant quelques secondes. En France, le Tokamak Tore-Supra, a confiné un plasma pendant six minutes, grâce à ses bobinages supraconducteurs et à une gestion efficace des flux de chaleur, justifiant ainsi l'espoir de faire fonctionner ces machines en régime permanent.

Le LMJ résulte de trente années de recherches classifiées et ouvertes sur le confinement inertiel (chapitre 2). C'est l'autre voie proposée pour aboutir au contrôle de la fusion nucléaire. Elle consiste à créer, pendant un bref instant et dans une petite quantité de mélange DT, les conditions pour que la combustion thermonucléaire s'amorce et se développe sans chercher à la contrôler, jusqu'à épuisement du combustible. La température initiale nécessaire reste très élevée, supérieure à cinquante millions de degrés. Si la matière est assez dense pour freiner les noyaux d'hélium, produits avec une énergie cinétique de 3,5 MeV, afin qu'ils déposent leur énergie dans la matière, il suffira de déclencher les réactions dans une petite zone, qui chauffera le combustible voisin à la bonne température pour qu'il s'enflamme à son tour. La réaction peut alors se propager

\footnotetext{
${ }^{4}$ Les Tokamaks, initialement développés en URSS, sont des machines dans lesquelles un plasma torique est confiné par un champ magnétique dont une partie est générée par des aimants et le reste par un fort courant circulant dans le plasma.

${ }^{5}$ Cette puissante crête $n^{\prime}$ a été obtenue que pendant trois secondes. Dans des décharges de plus longue durée, le rapport n'a pas dépassé 0,2 .
} 
dans la masse avant la dissociation du combustible, que gouverne la seule inertie, en l'absence de confinement. Pour optimiser les conditions de ces réactions, le laser comprimera une cible à des densités de 100 à 1000 fois la densité de l'eau et en ménageant une petite zone à très haute température, appelée point chaud, qui va servir d'allumette pour déclencher la combustion.

Pour y parvenir, le rayonnement du laser est dirigé sur une petite cible sphérique, constituée d'une coquille de DT solide cryogénique, à l'intérieur de laquelle subsiste un peu de combustible en phase gazeuse. L'irradiation de la cible provoque un chauffage violent de sa surface extérieure qui se trouve soumise à une très haute pression. Le matériau de surface se détend à grande vitesse dans le vide, en entrainant une ablation partielle de matériau. Par un effet analogue à celui qui, dans une fusée, permet aux gaz brûlés d'exercer une poussée, l'éjection de matière propulse la coquille vers le centre de la sphère, et la cible implose en comprimant le combustible qu'elle contient. La chronologie de l'implosion est réglée pour que la compression de la cible, sans subir trop de freinage, porte le gaz à une température suffisante afin que la fusion s'y déclenche, amorçant ainsi la combustion décrite plus haut, qui a reçu le nom d'ignition. L'ignition $n^{\prime} a$ jamais encore été réalisée en laboratoire. Le principe en a été cependant vérifié dans les expériences américaines "Centurion-Halite », où le rayonnement d'un engin nucléaire se substituait au laser pour faire imploser la cible. Les expériences de laboratoire ont permis, quant à elles, de déterminer les conditions précises pour lesquelles l'ignition se produit et d'identifier les contraintes à imposer à la fabrication des cibles pour minimiser l'énergie nécessaire du laser.

\section{3 Évolution des recherches dans les vingt prochaines années}

\subsection{Le confinement magnétique : Iter}

L'objectif de la machine lter est de parvenir à créer un plasma en combustion (chapitre 1). On entend par là un plasma dont la température est maintenue essentiellement par les réactions de fusion elle-même. La fusion du deutérium et du tritium libère un neutron de $14 \mathrm{MeV}$ et un noyau d'hélium de 3,5 MeV. Le neutron s'échappe mais le noyau d'hélium, grâce à sa charge électrique, est capturé par le champ magnétique de la configuration et cède son énergie au plasma, soit $20 \%$ de l'énergie totale libérée, réduisant d'autant la puissance à injecter pour compenser les pertes et maintenir la température au niveau nécessaire. Dans un réacteur rentable, l'énergie injectée ne devrait pas dépasser quelques pourcents de l'énergie produite. L'expérience lter ouvrira la voie dans 
cette direction et d'autres machines devront lui succéder pour aboutir à une véritable usine de production.

Dans les expériences menées jusqu'à présent, le chauffage du plasma par les noyaux d'hélium restait négligeable et la température ne se maintenait que grâce à une énergique injection de particules et de rayonnement. Dans le cas $d^{\prime}$ Iter, les performances nominales visent à produire un plasma où la puissance injectée ne représentera plus que la moitié de la puissance fournie au plasma par les noyaux d'hélium, soit $10 \%$ de la puissance totale de fusion. Le défi est de parvenir au contrôle des mécanismes physiques régissant ce plasma, dominé par les réactions de fusion, pendant 400 secondes, dans un volume de $840 \mathrm{~m}^{3}$. La maîtrise d'un plasma en combustion est un point de passage obligé sur la route du réacteur et l'obtention de ces performances représenterait une avancée incontestable vers la réalisation d'un réacteur. Dans ces conditions physiques nouvelles, le comportement du plasma guidera les choix de l'étape suivante.

Outre l'objectif de ces performances nominales, le programme expérimental $d^{\prime}$ Iter prévoit une deuxième phase $d^{\prime}$ exploitation, dite " avancée », qui permettra d'explorer des domaines plus proches du réacteur. Pour qu'une machine mérite le nom de réacteur, elle doit fonctionner en régime permanent, pendant des années, en produisant assez d'énergie pour compenser la consommation des auxiliaires indispensables et alimenter les turbines électrogènes à vapeur. La machine Iter n'atteindra pas ces régimes qui resteront l'apanage des dispositifs suivants, chargés d'en démontrer la faisabilité et déjà baptisés Demo. Mais Iter devra explorer les voies qui y mènent. Dans ce programme avancé, il ne suffira plus de se limiter seulement à des situations obtenues par une extrapolation raisonnable des machines précédentes, mais il faudra appréhender les limites et contours des différentes conditions de fonctionnement intéressant. En particulier, la durée du plasma sera allongée afin de sonder les possibilités de travail en mode permanent, des plasmas à plus fortes pressions mettront à l'épreuve la résistance de la configuration magnétique, des modifications fines du champ magnétique permettront d'explorer des configurations où ses propriétés isolantes s'amélioreront. II n'est pas exclu, si le succès accompagne ces tentatives, d'aborder le thème du contrôle d'un plasma à l'ignition, c'est-à-dire $d^{\prime}$ un plasma qui génèrerait par lui-même assez d'énergie pour assurer le remplacement des pertes et la génération du courant; la principale variable de contrôle deviendrait l'alimentation en combustible, au lieu de l'injection d'énergie, comme c'est le cas actuellement.

La réalisation de ces programmes validerait le concept de confinement magnétique sur le plan scientifique et introniserait les tokamaks comme principe de base pour un réacteur. C'est le socle de l'argumentation justifiant l'expérience Iter. 
À ces finalités, de nature plutôt fondamentale, vient s'ajouter un thème plus proche de l'ingénierie nouvelle qui accompagnera nécessairement l'exploitation industrielle de la fusion. Le succès ou l'échec de cette partie du programme n'engagerait pas la validité du confinement magnétique mais concernerait seulement la durée du travail restant à effectuer avant de pouvoir exploiter cette énergie de manière rentable. Il s'agit d'abord d'évaluer la résistance des parois proches du plasma aux conditions très dures créées par la proximité du plasma. Quant aux particules qui s'en échappent, elles seront conduites par le champ magnétique vers un dispositif périphérique, le divertor, où elles seront refroidies, neutralisées et pompées à l'extérieur du système. Ce divertor jouera aussi un rôle central dans l'évacuation des combustibles brûlés, dont l'étude fait partie du programme expérimental d'Iter. Un tel système fonctionne déjà sur le Jet. Mais, dans Iter, les flux thermiques plus élevés créeront des conditions plus sévères et feront de sa réalisation et de son endurance un des défis les plus difficiles à relever. De sa réussite dépendra la faisabilité des étapes suivantes.

La résolution de ces problèmes techniques conditionne la réussite du programme scientifique et ces recherches apporteront aussi de précieux enseignements pour les développements postérieurs à Iter. Mais, dans le cahier des charges de cette machine, les performances demandées se limitent à ce qui est nécessaire pour qu'lter fonctionne correctement et réponde aux questions concernant la faisabilité et l'intérêt de la fusion. Il en est de même pour l'ingénierie nucléaire. Les aimants et les équipements seront protégés des neutrons par des écrans bien dimensionnés, mais la quantité totale de neutrons émis pendant sa durée de vie ne justifie pas l'emploi de matériaux spéciaux résistant à une irradiation permanente très intense pendant des années, comme il le faudrait dans un réacteur. Dans ces écrans, l'énergie déposée par les neutrons sera évacuée vers l'extérieur pour limiter la montée en température des matériaux, au lieu de générer de la vapeur destinée aux turboalternateurs, comme devrait le faire un réacteur électrogène. De même, le tritium ne sera pas régénéré dans la machine, qui sera alimentée par le stock existant. Seuls des modules tests de dimensions significatives seront introduits localement pour mettre à l'épreuve des principes élaborés en prévision des autres étapes de la fusion. En ce sens, Iter sera loin de remplir toutes les fonctions primaires demandées à un réacteur dans l'industrie nucléaire. Il ne s'agit encore que d'une expérience de principe.

\subsection{Le confinement inertiel : LMJ}

Le LMJ a pour objectif scientifique de réaliser l'ignition d'une cible en la comprimant et en parvenant à créer le point chaud qui provoquera l'inflammation du DT cryogénique (chapitre 2). Cette installation, comme son homologue américaine le NIF, est financée essentiellement par la défense, dans le cadre d'un 
programme de simulation qui fait suite à l'arrêt des essais nucléaires. Mais l'objectif scientifique du LMJ coïncide avec la première étape $d^{\prime}$ 'un programme axé sur la production d'énergie à des fins civiles. En conséquence, une communauté civile, collaborant avec les scientifiques des laboratoires de défense, s'est constituée et s'est donnée pour programme l'utilisation des installations du LMJ et de ses dépendances pour avancer dans cette voie et défricher ce champ inexploré qui constitue une discipline émergente.

Les expériences passées ont validé des schémas de compression par ablation de matière et il reste à vérifier que ces résultats s'extrapolent bien pour les paramètres du LMJ. Mais, jusqu'à présent, aucune expérience de laboratoire $\mathrm{n}^{\prime}$ a pu créer le point chaud où seraient réunies les conditions de température et de densité demandées pour déclencher la combustion ${ }^{6}$. Le LMJ est dimensionné pour atteindre cet objectif et produire dix fois plus d'énergie que le laser $n^{\prime}$ en a injecté dans le combustible. Mais, à la différence d'Iter, l'énergie de fusion restera très inférieure à l'énergie dépensée pour générer l'énergie rayonnée par le laser, en raison du rendement très faible du laser lui-même. La justification première de l'expérience n'est pas la production d'énergie, mais la réalisation $d^{\prime}$ 'un banc d'essai pour mettre à l'épreuve les connaissances sur la dynamique de la combustion nucléaire. Pour que les futurs résultats s'intègrent dans une recherche de nouvelles sources d'énergie, le rendement du laser doit progresser de manière très importante, et $c^{\prime}$ est bien ce qui est entrepris, à une échelle encore assez modeste, en France et aux États-Unis, dans un cadre assez large pour que les étapes intermédiaires puissent être utiles à d'autres applications.

\section{Les verrous scientifiques et techniques pour Iter et le LMJ}

Avant d'envisager les solutions qui conduiront à une possible exploitation industrielle de la fusion, les installations en construction auront à démontrer la faisabilité d'une production d'énergie à partir de la fusion nucléaire, avec des rendements, des dimensionnements et des conditions de fonctionnement compatibles avec l'objectif final. Malgré les succès des machines en service actuellement, il subsiste des difficultés et des verrous qu'il convient d'évaluer, avant de proclamer la fin des investigations scientifiques et la venue de l'exploration technologique.

Dans le cas d'Iter et plus généralement dans celui des Tokamaks, le verrou principal reste l'optimisation de la stabilité et du confinement (chapitre 3). Les écarts à l'équilibre thermodynamique conduisent en effet au développement

\footnotetext{
${ }^{6} \mathrm{Si}$ on excepte les expériences baptisées Centurion-Halite, citées plus haut.
} 
d'instabilités dans une large gamme d'échelles spatiales et temporelles. Les instabilités de grandes échelles spatiales déforment le plasma et peuvent conduire à la perte du confinement. La compréhension et le contrôle de leur évolution ont déjà beaucoup progressé et ont permis de délimiter les domaines où elles restent inoffensives. Pratiquement impossibles à éviter, les instabilités à petite échelle évolvent vers un régime turbulent qui réduit la qualité du confinement en accélérant le transport des particules et de la chaleur vers les parois. Cette turbulence est associée à des structures spatiotemporelles aléatoires dont la description et la prévision font partie des questions non résolues de la physique.

Un second verrou est celui de l'interaction plasma-paroi (chapitre 7). En effet, les éléments de première paroi sont censés jouer des rôles multiples : extraction de la puissance, pompage des cendres $(\mathrm{He})$, contrôle de l'influx d'impuretés et alimentation en matière du plasma. La difficulté vient ici des processus complexes de physique atomique et de physicochimie qui interviennent dans la mise en œuvre de ces fonctions. Le divertor est au cœur de cette problématique. Le régime de fonctionnement choisi pour Iter assure un confinement optimal, qui, en contrepartie, entraîne de fortes variations de pression au bord du plasma. Les expériences sur le Jet ont montré que des instabilités résiduelles pouvaient se développer dans cette zone, avec des risques d'injection de plasma risquant $\mathrm{d}^{\prime}$ endommager le divertor ${ }^{7}$. Le contrôle de ce phénomène constitue un verrou qui peut limiter les performances et qui donne une importance toute particulière aux études actuelles sur ce sujet.

La modélisation numérique rend de nombreux services et intervient à tous les stades de l'exploitation et de l'interprétation des expériences ainsi que dans I'élaboration des théories (chapitre 6). Mais les performances complètes ne peuvent pas encore faire l'objet d'une prédiction par le calcul, car, comme prévu par les mathématiques, la turbulence introduit une variété de comportements très difficile à prendre en compte. Outre les problèmes fondamentaux introduits par la dynamique chaotique, les ordinateurs actuels ne peuvent pas encore traiter la grande disparité des échelles spatiotemporelles. Cette restriction constitue un véritable verrou en limitant la compréhension des observations et les explorations théoriques en dehors des voies tracées par les expériences existantes. L'amélioration des outils numériques constitue peut-être le défi le plus important avec comme objectif final l'analyse prédictive. Une telle situation n'est pas sans rappeler le problème des climatologues. La modélisation numérique jouera donc un rôle croissant, même si l'objectif initial d'un calcul complet n'est pas atteint.

\footnotetext{
${ }^{7}$ Sur le Jet, le divertor initial a été remplacé par un divertor pompé, afin d'assurer un meilleur contrôle de la densité. En fait, le régime de confinement optimal a relégué ce problème au second plan en raison de la présence de ces oscillations du bord du plasma.
} 
À défaut de simulations numériques complètes, les performances sont actuellement prédites à l'aide de lois d'échelle. Cette démarche empirique a été utilisée avec succès, grâce à une collaboration internationale remarquable qui a permis la mise en commun de tous les résultats obtenus sur les machines actuelles (voir chapitre $3 \S 3.1$ ). Cette méthode a pu être fiabilisée en utilisant un principe de similitude, fondé sur une expression adimensionnelle de ces lois d'échelles. On peut ainsi extrapoler vers Iter les résultats obtenus sur des machines existantes comme le Jet ou JT-60, une machine japonaise équivalente. L'essentiel du programme actuel de ces machines consiste à rechercher des conditions de plasmas stables et faiblement turbulents, qui sont ainsi transposables à lter. C'est dans cet axe que les progrès les plus importants sont attendus. Cette stratégie repose aussi sur la mise au point de diagnostics permettant de mesurer les propriétés d'un plasma de fusion.

Parallèlement à Iter, installation nucléaire performante aux procédures rigides, il sera important de conserver une activité de recherche sur des appareils plus modestes, aux performances moindres mais présentant une plus grande flexibilité. Il sera ainsi possible de faire progresser plus rapidement certains développements accompagnant l'exploitation d'Iter voire de générer des idées nouvelles qui pourraient alors être transposées sur Iter. Cette pratique s'est souvent avérée fructueuse dans le passé : le concept de divertor inventé dans le petit Tokamak allemand ASDEX a été implanté avec succès dans Jet qui n'incluait pas ce concept à l'origine ; les dispositifs de chauffage du plasma de Jet ont tous été étudiés et développés sur des installations de petite taille, plus accessibles et plus souples d'utilisation.

Dans le cas du LMJ, les instabilités jouent aussi un rôle crucial dans la limitation des performances et elles portent la responsabilité du gigantisme de l'installation. Elles ont ruiné les espoirs prématurés mis dans le confinement inertiel, dans les années 1970. Aujourd'hui, grâce à la théorie, à l'expérience et à la simulation numérique, les progrès sont considérables. II reste que la simulation numérique ne peut pas encore prendre en compte toutes les échelles spatiales et temporelles et que, là aussi, cet outil jouera un rôle central. L'observation pose des problèmes spécifiques liés aux dimensions minuscules de la cible après compression, au caractère hautement transitoire de l'implosion et de la combustion ainsi qu'aux conditions de densité et de température, gênant la propagation des rayonnements. Des méthodes nouvelles font leur apparition comme l'imagerie neutronique ou le laser à rayons $X$. Dans ce domaine aussi, la vérification de la validité des simulations numériques se situe au centre du programme de recherche et demande une très haute sophistication dans les diagnostics. 


\section{Les verrous techniques et la préparation de l'industrialisation}

Dès les origines, partisans et adversaires de la fusion s'accordaient sur un point : démontrer la faisabilité de la fusion contrôlée en laboratoire n'apportait pas la preuve qu'elle pouvait devenir une source d'énergie exploitable industriellement. Pour apporter cette preuve, il manquait un développement scientifique et technologique spécifique qui, s'il n'apparaissait pas totalement hors d'atteinte, demanderait des efforts longs et coûteux après la validation scientifique. Aujourd'hui, bien qu'il subsiste des zones d'incertitude sur l'extrapolation des résultats actuels à un réacteur, la démonstration scientifique de faisabilité est presque acquise et sera précisée par les expériences des prochaines années. De plus, le passage à des machines réellement thermonucléaires demande des investissements en forte croissance qui ne vont plus pouvoir se justifier en l'absence de la validation technologique. Le volet technique de la fusion doit donc être abordé, en se concentrant sur les aspects qui ne dépendent pas de la conjoncture actuelle et qui ont de bonnes chances de conserver une valeur dans les décennies à venir (chapitre 9).

Les deux grands projets, Iter et LMJ, s'appuient sur des technologies éprouvées, dont plusieurs ont fait récemment l'objet d'avancées importantes. Les points technologiques les plus sensibles concernent les matériaux faisant face au plasma et leur assemblage, dans Iter, et la tenue des optiques à haut flux pour le LMJ.

Pour le confinement magnétique, les principaux sous-ensembles d'Iter ont été testés avec succès. Par conséquent, les verrous technologiques se manifesteront surtout après Iter, en vue de la machine suivante, appelée Demo et plus tard lorsqu'il faudra passer au stade de l'industrialisation et de la rentabilité économique dans plusieurs dizaines d'années. Les questions les plus évidentes concerneront la transformation de l'énergie des neutrons en chaleur utilisable et sa récupération, la couverture tritigène, la longévité des matériaux de structure sous l'irradiation accumulée des neutrons de $14 \mathrm{MeV}$, la minimisation de l'activation des matériaux, l'optimisation du cycle du tritium.

En ce qui concerne les matériaux, la mise au point des réacteurs à fission a déjà conduit à aborder et résoudre des problèmes de résistance aux radiations (chapitre 8). Cependant, la fusion s'en distingue par quelques caractères importants et inédits. Au contraire de la fission où l'essentiel de l'énergie libérée se retrouve dans des noyaux lourds et chargés, les réactions de fusion produisent des neutrons très énergétiques ( $14 \mathrm{MeV}$ ) qui emportent $80 \%$ de l'énergie. Leur thermalisation va demander de fortes épaisseurs de matière sans atteindre des 
volumes prohibitifs, mais, surtout, leur haute énergie provoquera en abondance des réactions nucléaires générant des particules alpha ou de l'hydrogène qui altèrent les propriétés mécaniques en s'accumulant sur de longues périodes. L'exploitation industrielle de la fusion demande des solutions spécifiques à ces difficultés. Elles relèvent de la recherche en métallurgie nucléaire et nécessitent des expériences explicites sur les matériaux, expériences à ce jour non disponibles. Des travaux européens et américains ont déjà conduit à ce qui ne constitue encore que des pistes intéressantes, laissant penser que ces solutions existent, mais il reste à trouver l'alliage, le composite ou la céramique réunissant l'ensemble des qualités requises. Il faut considérer les défis (expérimentaux, théoriques, de modélisation) liés à cette situation nouvelle, comme un moteur du développement de la science des matériaux. En les abordant dès maintenant avec une ampleur suffisante (la mise au point d'un matériau nouveau est un long processus), on assurera une mobilisation des chercheurs de qualité qui assureront et perpétueront l'expertise indispensable tout au long de cette entreprise.

Les verrous techniques du confinement inertiel ont des similitudes avec ceux du confinement magnétique dans le domaine des matériaux et des couvertures tritigènes. En revanche, les différences sont notables dans le domaine des cibles et des sources d'énergie. La synergie la plus évidente avec le confinement magnétique concerne le comportement des matériaux de structure qui constitueront la chambre de réaction; signalons cependant la difficulté supplémentaire de l'émission de neutrons sous forme de flux pulsés extrêmement intenses, pour lesquelles il faudra probablement attendre la réalisation du réacteur pour entièrement valider les matériaux.

Le processus de fabrication de la cible cryogénique de deuterium-tritium sera mis au point pour les expériences avec le LMJ. Mais, le passage au stade du réacteur, qui fonctionnera à une cadence $\mathrm{d}$ 'injection de quelques hertz, impliquera des ruptures technologiques sur les procédés de conception et de fabrication des cibles afin de baisser considérablement leur coût. En ce qui concerne la source d'énergie, d'une part, elle devra permettre plusieurs implosions par seconde alors que le LMJ n'en réalisera que quelques unes par jours, et, $d^{\prime}$ autre part, le rendement de la source devra progresser $d^{\prime}$ 'au moins un ordre de grandeur. Ces deux problèmes constituent des obstacles difficiles à surmonter et pour lesquels il n'existe pas encore de solution disponible.

\section{6 | Les autres filières ou solutions innovantes}

Dans le domaine du confinement magnétique, le Tokamak est considéré sans conteste comme la configuration susceptible de mener le plus rapidement au confinement d'un plasma thermonucléaire, dans des conditions représentatives 
de celles qui sont prévues dans un réacteur producteur d'électricité. Pour faire d'un tokamak un véritable réacteur, il reste à franchir des étapes importantes, et, comme déjà souligné, la certitude de la faisabilité reste encore à prouver. Un des problèmes les plus importants concerne la stationnarité du fonctionnement qui semble difficile à contourner et nécessite le maintien du courant interne par un important système auxiliaire. Le caractère très compact de certains composants internes peut aussi soulever des objections de la part des exploitants.

Le stellarator, une configuration toroïdale sans courant interne, offre une solution puisque le confinement du plasma ne dépend que de champs créés par des aimants extérieurs. La stationnarité est inhérente à la configuration. De plus, en principe, une certaine liberté subsiste dans le choix des paramètres géométriques. En revanche, cette configuration perd la symétrie cylindrique du Tokamak, ce qui conduit à un coût élevé pour la fabrication des bobines et à un contrôle plus difficile du transport de l'énergie et des particules. Les machines actuelles en service sont de plus petites dimensions que le Jet, mais elles démontrent déjà que les lois de transport ne diffèrent pas fondamentalement de celles des Tokamaks. Au cas où il serait nécessaire de choisir cette configuration, l'expérience acquise sur les Tokamaks ne serait pas perdue pour autant car les résultats acquis sur le chauffage du plasma, l'interaction du plasma avec les parois, l'évacuation de l'hélium et la réduction des pertes seraient rapidement transposés aux stellarators.

Les travaux sur le confinement inertiel n'ont pas encore débouché sur une machine extrapolable jusqu'au réacteur. Le rayonnement laser remplit les conditions de souplesse et de précision demandées par l'implosion d'une cible, mais le taux de répétition comme le rendement ont d'énormes progrès à accomplir. Les accélérateurs d'ions lourds pourraient prendre le relais, mais il n'existe encore aucune donnée expérimentale permettant de jauger leurs capacités réelles. Ils présenteraient l'avantage de n'avoir aucun des deux handicaps des lasers. Mais, ils manquent de souplesse, de précision et nécessitent un investissement financier très important.

Aussi, les réflexions actuelles sur les prototypes de réacteurs industriels sont plutôt conçues avec un laser comme source d'énergie pour le confinement inertiel, ce qui implique des développements importants sur des nouvelles générations de lasers (solide pompés par diodes, lasers UV).

Une variante récente et innovante du confinement inertiel consiste à utiliser deux types de laser, l'un pour comprimer la matière, l'autre, très intense dans le domaine des petawatts, pour créer le point chaud en fin de compression (chapitre 4). L'étude de l'efficacité de cette méthode et sa mise en applications impliquent l'exploration de phénomènes qui certes peuvent se produire dans I'Univers, mais dont l'accessibilité enfin possible en laboratoire ouvre un tout 
nouveau domaine de la physique des plasmas. L'avantage essentiel de la nouvelle méthode réside dans la dissociation totale de la compression et du chauffage. Dans la méthode traditionnelle, le point chaud se forme au centre de la cible et le laser doit assurer simultanément la compression du combustible cryogénique en créant le minimum d'entropie et le chauffage du point central à des températures de l'ordre de cinquante millions de degrés.

La faisabilité de ce nouveau schéma, baptisé ignition rapide, attend encore une démonstration probante. Plusieurs solutions se présentent aujourd'hui : toutes s'appuient sur un laser de haute énergie et de très forte puissance, dans la gamme des petawatts, chargé de délivrer en un temps très court l'énergie nécessaire pour porter une petite région de la cible comprimée à la bonne température, avant qu'elle ne se dissocie. Le dépôt de l'énergie dans le plasma et le transfert de cette énergie au cœur de la cible pose des problèmes de physique des plasmas originaux qui enrichiront la discipline. En ce qui concerne I'application à la fusion, la réussite de la méthode, en abaissant les contraintes en énergie des lasers et en symétrie de la compression, feraient probablement entrer le confinement inertiel dans le domaine des solutions crédibles pour la production d'énergie.

\section{Organisation de la recherche en fusion : aspects nationaux, européens et internationaux}

\subsection{La recherche de base}

La fusion, comme toute grande avancée technologique, fera appel à un grand nombre de domaines de la physique et de la technique. La physique nucléaire fournira ses méthodes de détection spécifiques et ses outils d'interprétation, I'hydrodynamique interviendra aussi bien dans compréhension des mouvements du plasma que dans les écoulements des fluides extracteurs et caloporteurs, la physicochimie des surfaces permettra de mieux assurer la coexistence du plasma et des parois, la métallurgie élaborera des matériaux résistants aux neutrons, l'optique apportera ses diagnostiques et ses lasers (chapitre 5). L'électrotechnique dominera la conception et le pilotage des machines; dans les bobinages supraconducteurs et les cibles laser, la cryogénie cohabitera avec les millions de degrés; le génie nucléaire jouera son rôle comme il doit le faire dans une telle installation; la robotique sera partout présente.

Mais la physique des plasmas chauds restera encore longtemps au cœur du problème et ses avancées éclaireront les phénomènes complexes qui se produisent dans les plasmas thermonucléaires (chapitres 3 et 4). Les instabilités, la 
turbulence et le transport associé ont fait de grand progrès, mais ils sont loin d'être véritablement compris, d'autant que les régimes explorés par lter et le LMJ relèvent $d^{\prime}$ une physique originale qui ne se réduit pas à la mécanique des fluides, suffisante le plus souvent jusqu'à présent. Dans ces futures machines, l'assimilation du plasma à un fluide conducteur ne permet plus de prendre en compte correctement l'évolution du milieu. Bien que les mouvements apparents soient macroscopiques, il est nécessaire de décrire les orbites des particules individuelles dans les champs imposés de l'extérieur ou générés par le plasma lui-même. Il s'agit là d'un programme scientifique de grande ampleur. Cette thématique est à l'interface entre plusieurs communautés, notamment les mathématiques appliquées, l'astrophysique et la physique des plasmas de fusion. La modélisation de cette turbulence requiert un effort vigoureux dans le domaine des simulations numériques. Mais le seul calcul numérique ne suffira pas pour résoudre tous les problèmes, car les ordinateurs actuels ne parviennent pas encore à décrire ces situations $a b$ initio, et, même s'ils y parvenaient, ils seraient incapables de proposer des améliorations et de forger de nouveaux concepts. Les théoriciens des plasmas doivent aussi avoir une place importante dans la fusion, afin d'assurer une production scientifique de qualité autour de ces installations coûteuses et afin de centrer les recherches sur les aspects les plus profonds et les plus universels.

\subsection{L'organisation de la recherche sur le confinement magnétique}

\subsubsection{Le contexte européen}

L'activité de recherche européenne en fusion par confinement magnétique est fortement intégrée, notamment via une structure en associations entre laboratoires nationaux et l'Euratom. II n'y a pas de programme national au sens strict du terme mais un programme européen exécuté par un ensemble d'instituts qui s'engagent contractuellement avec l'Euratom. Les différentes parties du programme sont complémentaires : au Jet l'étude des performances, à Tore Supra la physique et la technologie du fonctionnement en continu. Les grandes lignes programmatiques, le budget et l'exécution des travaux des instituts associés (communément appelés associations pour la fusion) sont contrôlés par un Comité de gestion mixte comprenant quatre membres de la Commission et quatre membres du laboratoire de recherches. Il y a actuellement plus de vingt instituts de recherches impliqués dans le programme Fusion européen. Tous les grands pays de l'Union européenne et la Suisse ont signé des contrats d'associations. En France, le CEA est le dépositaire de ce contrat et a donc la charge de la coordination des activités françaises (signature du contrat Euratom dès 1958). 


\subsubsection{Le contexte hors Europe}

Pour les collaborations hors Europe, l'Euratom élabore des accords de coopération bilatéraux qui sont les canaux privilégiés pour les associations impliquées dans le programme fusion européen et les instituts de recherches hors Europe. En particulier, elle a établi des collaborations globales dans le cadre de l'AIEA (Agence internationale de l'énergie atomique) ou de l'AIE (Agence internationale de l'énergie).

L'AIEA a très tôt favorisé les échanges dans le domaine de la fusion. Ainsi, le développement d'un réacteur thermonucléaire a débuté sous les auspices de I'AIEA en 1978 sous le nom d'Intor (International Toroidal Reactor) et c'est tout naturellement que I'AIEA fournit le cadre légal nécessaire au développement d'Iter de 1988 à nos jours. Le développement de la base internationale de données du confinement des Tokamaks, capitale pour les extrapolations des futures machines et d'Iter en particulier, se déroule aussi dans ce cadre (International Tokamak Physics Activity).

Un comité de recherche, I'International Fusion Research Council (IFRC) est chargé de conseiller le directeur de l'AIEA sur les programmes et collaborations que l'AIEA peut soutenir. Les collaborations sont formalisées sous la forme de "Coordinated Research Projects, CRPs ».

L'AIEA organise annuellement une série de réunions techniques sur des sujets précis et tous les deux ans une conférence regroupant près de 600 chercheurs (AIEA Fusion Energy Conference). L'AIEA est aussi responsable de la publication du journal « Nuclear Fusion » couvrant les travaux menés pour la fusion magnétique et inertielle.

L'AIE procure un autre cadre pour le support des collaborations internationales. Elles sont formalisées par l'émission d'un "Implementing Agreement » contractualisant les relations entre au moins deux membres de l'AIE. Un comité spécifique, Committee on Energy Research and Technology (CERT) valide ces collaborations, réparties en cinq principaux programmes : combustibles fossiles, énergies renouvelables et la production d'hydrogène, l'utilisation finale de l'énergie, la fusion, les sources d'informations sur l'énergie.

\subsubsection{Organisation en France}

Le CEA conduit des recherches sur la fusion contrôlée par confinement magnétique pratiquement depuis sa création. Ces études se réalisent dans le cadre de l'association Euratom-CEA dont la gestion est confiée au Département de recherches sur la fusion contrôlée (DRFC), qui fait partie de la Direction des 
sciences de la matière (DSM) du CEA. À ce titre, le DRFC fait office de point d'entrée français au programme Euratom sur la fusion magnétique et assure vis-à-vis de la Commission, la gestion, la coordination et la responsabilité des études qui sont confiées à l'association. Les activités scientifiques de l'association consistent principalement à exploiter le Tokamak Tore Supra (Cadarache), à participer à l'ensemble du programme européen sur la fusion, notamment l'exploitation du Tokamak européen Jet (Joint European Torus) ainsi que les développements des technologies nécessaires aux prochaines machines de fusion, et à préparer la venue d'Iter à Cadarache. Ces activités scientifiques intègrent des contributions d'autres laboratoires (CEA mais aussi CNRS, universités, industries) sur une thématique large, qui inclut notamment les questions liées à la physique des plasmas de fusion, aux matériaux, aux aimants supraconducteurs, à la sûreté, à la conception et à la maintenance des futures installations de fusion. Ces activités se prolongent au travers de collaborations avec des laboratoires étrangers et nationaux, dans le cadre des institutions (Europe et hors Europe).

Comme la plupart des départements du CEA, le DRFC réunit dans un même lieu ingénieurs et physiciens. Cette structure permet de couvrir un spectre d'activités très large qui recouvre la technologie des Tokamaks, le développement de diagnostics, et la physique des plasmas de fusion. Cette spécificité est un atout indéniable pour l'accueil d'Iter à Cadarache.

L'Association Euratom-CEA participe activement à l'ensemble du programme européen et est en particulier très impliquée dans l'exploitation du Tokamak européen Jet, situé à Culham (Royaume-Uni). L'Association Euratom-CEA contribue aussi fortement aux développements des technologies nécessaires aux futures machines de fusion. Les contributions de l'association Euratom-CEA représentent environ $20 \%$ de l'activité européenne dans ce secteur et couvrent en particulier le domaine des grandes bobines supraconductrices, des composants faisant face au plasma (tenue aux flux de chaleur), des matériaux de structure (tenue aux neutrons de $14 \mathrm{MeV}$ ), de la maintenance robotisée, de la sûreté, des composants produisant in situ le tritium. Ces contributions sont en majorité effectuées via des collaborations contractualisées avec des laboratoires hors DRFC comme des départements du CEA ou l'industrie. À ce titre, l'apport et l'intégration des compétences (nucléaires et hors nucléaires) des départements du CEA confèrent à l'Association Euratom-CEA la caractéristique unique en Europe de pouvoir appréhender dans sa globalité les thématiques posées par la fusion magnétique. Les collaborations entre l'association Euratom-CEA, le CNRS et les universités sont structurées autour de laboratoires de recherche conventionnés (LRC) et de cinq axes majeurs de recherches : théorie (gyrocinétique, transport, équilibre), études expérimentales (turbulence de centre et de bord), interaction plasma-paroi, physique atomique, et ondes. Les LRC et les contrats EuratomCEA correspondent à 37 chercheurs et ingénieurs permanents impliqués, dans 
I'activité Fusion/Énergie pour l'année 2003. Cet effectif augmentera en 2005 d'une quinzaine de chercheurs travaillant sur les questions de matériaux. Enfin une dizaine de physiciens travaillent sur des questions de physique amont.

\subsubsection{Organisation de l'enseignement}

Une évaluation des besoins pour lter et du programme d'accompagnement indique que la France devrait fournir de l'ordre de 500 ingénieurs et physiciens sur dix ans. Compte tenu des 225 experts existants et des départs en retraite prévisibles, la formation et le recrutement de 250 à 300 ingénieurs et physiciens des plasmas dans les dix ans à venir semble être une estimation raisonnable.

La formation de 300 ingénieurs et physiciens des plasmas sur dix ans pourrait passer par la création conjointe par les établissements d'enseignement supérieur disposant des compétences plasma d'une nouvelle spécialité "science de la fusion ". Cette fédération des formations aux sciences de la fusion intégrerait de façon cohérente les besoins de la fusion inertielle et magnétique. Il a donc été proposé de créer un master national "sciences de la fusion", couvrant l'ensemble des aspects de physique et de technologie en fusion magnétique et inertielle. Ce master serait implanté sur quatre sites universitaires (Aix-Marseille, Bordeaux, Paris, Nancy), et piloté conjointement pas les universités locales et I'Institut national des sciences et techniques nucléaires (INSTN). Cette initiative a par ailleurs reçu le soutien de plusieurs écoles d'ingénieurs. Un master a été créé le 17 mai 2006. Ce master pourrait s'intégrer dans une structure plus large de master européen, qui est en cours de discussion. L'encadrement de ce master combiné à l'effort de recherche nécessaire requiert un effectif en France d'environ 35 à 40 enseignants-chercheurs spécialisés.

La formation d'ingénieurs et technologues passe par une sensibilisation des écoles d'ingénieurs à la thématique fusion. Par ailleurs, la création d'un master piloté conjointement par I'Institut national des sciences et techniques nucléaires (INSTN) et l'Université permettrait de former des experts aux technologies associées à la fusion (supraconducteurs, électrotechnique, chauffages radiofréquence, matériaux, techniques nucléaires, ...)

\subsubsection{Organisation de la communauté scientifique}

Le développement d'un projet de l'envergure d'Iter, suppose l'appui et le renforcement des contributions de type amont. Les problèmes soulevés par les grands instruments que sont les Tokamaks sont fortement pluridisciplinaires. Ils requièrent autant de développements de haute technologie que d'études de physique fondamentale, dans lesquelles la communauté académique a toute sa 
place. La participation active du CNRS au projet lter implique une augmentation notable de l'effort de l'organisme, en synergie avec le CEA, porteur du projet au niveau national. La création d'une structure nationale permettrait de mieux intégrer l'organisation collective de l'activité de recherche et de formation. Elle viserait à garantir la cohérence de la politique de recherche, la synergie entre les différents partenaires. S'appuyant sur les politiques scientifiques propres de chacune des unités et leurs spécificités, elle donnerait à l'ensemble des laboratoires un poids et une visibilité significative au niveau international dans le projet Iter.

Cette structure nationale pourrait prendre la forme d'une fédération de recherche s'appuyant sur les laboratoires de recherche conventionnés. Elle pourrait prendre appui sur une unité mixte de recherche axée sur les aspects amonts et localisée à proximité d'Iter. La fonction principale de cette structure fédérative serait d'organiser la participation française à l'exploitation scientifique $\mathrm{d}^{\prime}$ Iter $^{8}$ D'autres équipes, déjà sous contrat Euratom-CEA ou intéressées par les thématiques développées, pourraient être associées. L'activité de la fédération autour des projets bénéficiant des financements Euratom serait pilotée par un conseil scientifique représenté au comité de gestion de l'association avec Euratom. Ce conseil assurerait la coordination scientifique, favorisant les collaborations, le relais des appels d'offre et la préparation des projets nouveaux dans le cadre international. La sélection des projets et l'évaluation seraient également assurées par le conseil, assisté d'experts.

Cette structure fédérative vise aussi à promouvoir les activités des différents groupes et élargir la communauté. Elle aurait aussi pour mission de coordonner et susciter les actions de formation. Elle permettrait aussi la valorisation de Tore Supra et du parc de petites machines existant des laboratoires de l'Université ou du CNRS. Des installations de taille raisonnable doivent être maintenues autour $\mathrm{d}^{\prime}$ Iter, tant dans le but de formation que pour faire progresser la physique de base et la mise au point des diagnostics. ${ }^{9}$

La part CNRS/Université de l'activité de recherche amont pourrait d'autre part bénéficier du soutien d'actions concertées du ministère de la Recherche, de I'ANR et du CNRS et participer aux programmes interdisciplinaires, comme le programme Énergie du CNRS. Un groupe d'analyse thématique (GAT) effectue depuis 2002 dans ce cadre une mission d'analyse et d'expertise. Des projets de

\footnotetext{
${ }^{8}$ Cette structure serait similaire à l'organisation mise en place pour l'exploitation du projet Laser Mégajoule : une fédération de laboratoires (Institut laser plasma) s'appuyant sur une UMR créée à Bordeaux (Celia).

${ }^{9}$ Une réflexion s'est engagée autour des moyens expérimentaux qui permettraient une approche globale des problèmes de la physique du plasma chaud confiné (MHD, turbulence et transport, interaction plasma-paroi et physique du divertor, spectroscopie, nouveaux diagnostics, ...), et qui répondent aux critères de formation, de cohérence et de pertinence du programme.
} 
recherche pourraient être développés également dans ce cadre programmatique à plus long terme.

Enfin, dans l'hypothèse d'une participation significative du CNRS et des universités à cette structure fédérative et à une unité mixte de recherche (UMR) localisée près d'Iter, une structure administrative pourrait être envisagée sur la base d'un élargissement de l'association Euratom-CEA.

L'institut fédératif devra faire face au problème du renouvellement, actuellement insuffisant, des équipes par de jeunes chercheurs. Ce problème est d'autant plus préoccupant qu'il est amplifié par le prochain départ en retraite de nombreux chercheurs et enseignants-chercheurs.

\subsection{L'organisation de la recherche pour le confinement inertiel}

Dans le domaine du confinement inertiel, la recherche s'est organisée en s'adaptant aux conditions particulières que lui ont imposées I'histoire et la nature du sujet. Depuis l'arrêt du laser Phébus, l'Europe ne disposait plus de grand laser de la classe demandée par la fusion. Une part importante des moyens intellectuels et numériques restait mobilisée sur le sujet afin de dimensionner le LMJ et de préparer son programme. Les autres chercheurs, essentiellement basés dans les laboratoires civils, ont poursuivi des études fondamentales liées à l'interaction des lasers à haut flux avec la matière. Une remarquable moisson de résultats très divers a démontré la richesse du domaine. Les travaux sur l'interaction laser-plasma ont connu de remarquables développements. Ils ont débouché sur des idées nouvelles pour mettre en œuvre la fusion par confinement inertiel avec le concept d'ignition rapide déjà évoqué. Ils ont fait considérablement progresser la compréhension de cette interaction dans les domaines d'utilisation pour le LMJ. Initialement prévu pour régulariser la distribution de l'intensité des faisceaux, le lissage optique a donné un outil efficace pour contrôler les instabilités liées à l'interaction. Les progrès dans l'accélération de particules chargées par laser ultra-intense ont partie liée avec toute cette activité.

Ces recherches, distribuées dans plusieurs laboratoires et équipes, ont toutes su développer de solides réseaux de collaborations, nationaux, européens et internationaux. Néanmoins, jusqu'à présent, cette collaboration était opérationnelle sur des installations laser de type académique, que l'on peut classer comme de "petits grands instruments", tel que le Luli à l'École polytechnique à Palaiseau. Le LMJ est d'une toute autre échelle pour permettre une démonstration de l'allumage d'une cible et l'adaptation de la communauté à ces nouvelles conditions ne se fera pas sans aide. La création d'une unité mixte de recherche impliquant le CEA, le CNRS et l'université de Bordeaux, traduit la volonté manifeste 
de ces organismes de rapprocher les diverses communautés autour des grandes installations en construction. Cette UMR regroupe les recherches qui concernent la fusion par confinement inertiel à des fins civiles, participer à la construction et la mise au point des installations ayant cet objectif et accueillir des études sur les retombées concernant d'autres domaines de la science. Par sa proximité du site, elle jouera également le rôle de point d'entrée préférentielle pour les équipes d'utilisateurs venant effectuer leurs expériences sur ces dispositifs.

En effet, programme de la défense, le LMJ va être ouvert au civil comme réaffirmé par exemple par le comité interministériel $d$ 'aménagement du territoire (CIADT) de décembre 2002 qui a créé le concept de Route des lasers, confirmé par le CIADT de décembre 2003 avec la décision de construction d'un laser petawatt ${ }^{10}$ sur la Ligne d'intégration laser (ou $\mathrm{LIL}^{11}$, prototype opérationnel du LMJ). Cette ouverture est déjà une réalité, mise en œuvre dans un premier stade sur la ligne d'intégration laser.

La réussite de l'ouverture scientifique du LMJ repose sur la création d'une communauté, motivée par les recherches sur la fusion à des fins civiles, utilisant les lasers et prête à s'investir dans ces programmes. La situation aujourd'hui présente des éléments encourageants, comme les créations de l'UMR de Bordeaux et de l'Institut laser et plasma, le soutien des pouvoirs publics ou l'intérêt international pour ces projets, mais il subsiste des difficultés préoccupantes comme la faiblesse numérique de la communauté impliquée, l'absence d'un dessein affiché au niveau du gouvernement, et les incertitudes liées aux questions de classification.

\subsubsection{L'Instifut laser et plasmas (ILP)}

Créé en mars 2003, I'Institut laser et plasmas (ILP) regroupe la communauté scientifique travaillant dans ces domaines. Il a pour mission d'élaborer un programme scientifique et technologique associé à une vision vers la fusion pour l'énergie, en s'appuyant sur l'UMR récemment créée à Bordeaux. II doit mobiliser des compétences adossées sur une recherche amont pour le LMJ et couplées aux installations lasers du milieu académique : étude des états extrêmes de la matière à haute température et densité, de la physique stellaire, applications des jets de particules relativistes engendrées dans l'interaction laser-matière, etc.

\footnotetext{
${ }^{10}$ Le laser petawatt délivrera une puissance crête supérieure à $10^{15}$ watts (un petawatt). II devrait générer une impulsion de $3,5 \mathrm{~kJ}$ en $10^{-12}$ seconde.

${ }^{11}$ La LIL, formée de quatre faisceaux colinéaires est le laser le plus énergétique d'Europe. II délivre $40 \mathrm{~kJ}$ dans I'UV en une dizaine de nanosecondes. En prenant le nom d'Octalil, il devrait voir le nombre de ses faisceaux doubler vers 2009 et les huit faisceaux éclater suivant une géométrie permettant la compression d'une cible. Le laser petawatt pourra alors se coupler à Octalil pour étudier l'ignition rapide.
} 
Cette organisation nationale rassemble près de 30 laboratoires et 250 scientifiques en France, sous la forme d'une fédération de recherche reconnue par le CNRS, le CEA, l'École polytechnique et l'université Bordeaux 1. Elle possède déjà une certaine visibilité internationale (appel d'offres international pour l'accès à des expériences sur la LIL en 2006, comité international pour la sélection des projets, organisation et présidence des grandes conférences du sujet). Elle accorde une aide pour concevoir, défendre et mener des projets complexes pour répondre à l'appel d'offres LIL 2006. Outre une politique de formation en liaison avec les formations pour Iter, elle assure la diffusion de la connaissance vers le grand public, en organisant des enseignements autour de thématiques telles que l'énergie, l'environnement, le développement durable... Une telle information largement diffusée sur les grands projets devrait ainsi attirer plus de jeunes vers des disciplines scientifiques où l'on convient que la taille de la communauté scientifique est sous-critique.

\subsubsection{Le support des pouvoirs publics}

L'État, des organismes et des établissements assurent en principe le financement et la pérennité de ce programme. Les décisions du CIADT de décembre 2002, prévoient des financements dédiés à la construction d'un bâtiment de I'ILP et à la construction du laser petawatt couplé à la LIL. La Région Aquitaine a financé des pré-études sur le laser petawatt et s'est portée maître d'ouvrage de sa construction avec un financement permettant de lancer la première phase du projet. Le CEA met à disposition du temps d'utilisation de la LIL pour la communauté académique dès 2005. Il est maître d'œuvre de la construction du laser petawatt qu'il réalise en partie avec des équipements identiques à ceux du LMJ et s'est engagé à financer son maintien en condition opérationnelle. L'université Bordeaux 1, membre fondateur de l'ILP, appuie les projets d'accompagnement de l'ouverture du LMJ par des recrutements fléchés «laser et fusion », par des actions fortes de recherche, de formation et le soutien aux infrastructures. L'ensemble de ces soutiens, s'ils perdurent, assoit l'Institut dans une position stable et manifeste l'intérêt soulevé par ces recherches dans des milieux scientifiques et techniques qui débordent largement le seul cadre de la fusion.

\subsubsection{L'organisation internationale}

Au plan international, deux organismes se sont intéressés depuis la fin des années cinquante à la fusion inertielle comme source d'énergie civile, mais aucune coopération internationale, comme celle qui existe dans le domaine magnétique, n'a été mise en place. 
D'une part, l'AIEA, sous la direction de l'International Fusion Research Council, a mené un programme d'échange d'informations sur les recherches autour de la production d'énergie par fusion inertielle (IFE). D'autre part, en 1998, un rapport, préparé à la demande du Comité consultatif pour le Programme Fusion de la Communauté européenne, montrait que le niveau global de compétences de l'Europe dans le domaine de la fusion inertielle était comparable à celui des États-Unis et qu'il était essentiel de se préparer par une concertation accrue à la démonstration en France, de la combustion du DT en laboratoire. Toutefois, dans ce domaine, la Communauté européenne se limite à une activité de veille technologique.

S'il est désormais reconnu qu'il est de l'intérêt de la France d'ouvrir les installations LIL et LMJ aux activités scientifiques civiles, il est tout aussi clair que le volet défense de l'activité $\mathrm{FCl}$ a été un frein à la collaboration scientifique internationale. Ceci est vrai, à la fois du côté des responsables des équipements de la défense pour qui les inconvénients l'emportaient sur les avantages, et du côté des scientifiques pour des raisons idéologiques compréhensibles et parfaitement respectables. Néanmoins, un projet européen d'une feuille de route vers la fusion inertielle par allumage rapide est en discussion actuellement, sous l'égide des physiciens anglais du Rutherford Appleton Laboratory. Dans ce contexte il est fondamental que la France convainque la communauté scientifique européenne de sa volonté irrévocable d'ouverture scientifique sans lien avec les activités de défense et avec une disponibilité suffisante des installations. L'ILP, structure purement académique, a été créé à cet effet, pour être le canal exclusif de l'ouverture civile. Une charte signée entre l'ILP et le CEA, et accessible sur le Web, définit les droits et devoirs des utilisateurs. Ainsi, un appel d'offres à projets sur la LIL pour l'année 2006 a été publié dans la presse scientifique internationale, dans le numéro de Physics Today de mai 2005. L'ouverture internationale se fera donc par étapes à partir de la réalisation des premières collaborations expérimentales sur la LIL.

\section{Conclusions et propositions}

Aboutissement de quarante ans de recherche en confinement magnétique, fruit d'une coopération internationale et européenne exceptionnelle, lter est un jalon scientifique et technique décisif vers l'exploitation de l'énergie de fusion. Le maintien d'une collaboration internationale la plus large possible fait partie des conditions indispensables à remplir pour assurer une qualité scientifique et technique à la hauteur des enjeux et au niveau de celle qui a conduit aux succès du passé, élargir le recrutement des personnels à une communauté suffisamment étendue et alléger la charge financière pour chacune des parties impliquées. 
La contribution française à l'exploitation scientifique de la machine devrait être préparée dès aujourd'hui en constituant un pôle scientifique " fusion magnétique » regroupant les efforts des organismes publics de recherche (physique et technologie) et en organisant les enseignements nécessaires à la formation des chercheurs, ingénieurs et techniciens indispensables à la réussite de ce programme. Dans ce contexte, il parait nécessaire de maintenir Tore Supra en activité comme base d'un programme d'accompagnement pour préparer la mise en service de la nouvelle machine.

Au niveau européen, la coordination qui a permis de placer l'Europe en tête des recherches sur la fusion par confinement magnétique doit être maintenue. Toutefois, il semble souhaitable d'assurer la dualité entre recherche et conduite de projet. Le renforcement des efforts de modélisation, dans un cadre européen est indispensable pour atteindre le niveau des USA et du Japon afin d'accompagner la démarche théorique qui doit elle-même être stimulée et encouragée dans le domaine de la turbulence et des instabilités en phase non linéaire. Cette démarche implique l'existence de moyens de calculs disposant d'une puissance et d'une disponibilité suffisantes, ainsi que la formation et le recrutement de chercheurs dans ces domaines afin que se forme en Europe une communauté de la modélisation "fusion », à la hauteur des enjeux et qui devrait être, en qualité et en volume, équivalente à celle des États-Unis ou du Japon.

En ce qui concerne les matériaux pour le réacteur, dans un premier temps, la simulation et la théorie devraient également être encouragées et organisées au niveau européen, en attendant des moyens expérimentaux dans le cadre de la collaboration internationale IFMIF (International Fusion Material Irradiation Facility). Les compétences de la communauté des matériaux pour la fission nucléaire devraient être fortement sollicitées. Il semble important de la rassembler dans une structure de projet, chargée du développement des matériaux résistants aux neutrons rapides. Il faut rappeler que la mise au point d'un matériau est toujours une affaire longue ( 15 à 20 ans), a fortiori dans le cas de la fusion où on se trouve dans des conditions inédites par la valeur élevée du rapport entre le taux de production d'hélium insoluble et le taux de production de défauts ponctuels par irradiation. Cependant, des progrès rapides sont attendus en matière de modélisation prédictive du comportement des matériaux dans ces conditions de fonctionnement tout à fait nouvelles.

La coordination de l'ensemble du programme fusion par confinement magnétique pourrait être efficacement développée dans un cadre international en s'appuyant sur deux pôles: Iter et l'exploitation commune des grands moyens expérimentaux. 
Pour la fusion par confinement inertiel, l'étape ultime de la démonstration de faisabilité de la $\mathrm{FCl}$, en réalisant la combustion du DT en laboratoire, est en cours.

Le contexte purement national du confinement inertiel a facilité la mise en place de structures efficaces de collaboration entre le CEA et les organismes de recherche civile. Cette situation doit être maintenue et encouragée. Elle constitue une expérience à laquelle la communauté de la fusion magnétique pourrait s'intéresser dans le cadre de la création du pôle scientifique mentionné précédemment. Il peut en résulter un rapprochement des deux communautés sur des questions communes comme l'enseignement, la conception du réacteur et les matériaux.

L'ouverture des installations LIL (et d'un laser petawatt qui lui sera couplé) et bientôt LMJ, constitue une opportunité unique pour la recherche, à la fois dans le domaine fondamental de la connaissance de la matière portée à des conditions extrêmes, pour des travaux prospectifs sur l'énergie et pour réaliser des diagnostics de plasmas en commun. Il est essentiel que cette volonté d'ouverture de la France, concrétisée par la création de l'ILP, bénéficie d'une promotion internationale importante. Par ailleurs, une concertation européenne dans le domaine de l'énergie par confinement inertiel apparaît maintenant pertinente ; il serait souhaitable en particulier de mener une réflexion sur la conception d'un réacteur viable et sur les actions à entreprendre pour progresser dans ce sens.

Finalement, il faut souligner la nécessité de créer un nouveau programme de formation aux sciences de la fusion, destiné aux scientifiques et aux techniciens qui seront amenés à exploiter, dans moins d'une dizaine d'années, Iter et le LMJ, deux complexes de recherche $d^{\prime}$ une envergure sans précédent que la France a entrepris de construire sur son sol.

Chapitre 1 - La filière Tokamak et la machine Iter

Chapitre 2 - La fusion par confinement inertiel

Chapitre 3 - Les plasmas chauds magnétisés

Chapitre 4 - Interaction laser-plasma et laser petawatt

Chapitre 5 - La physique atomique pour la fusion

Chapitre 6 - La simulation numérique

Chapitre 7 - Interaction plasma-paroi

Chapitre 8 - Les matériaux pour la fusion

Chapitre 9 - Les études de sûreté

Chapitre 10 - La fusion par confinement inertiel et l'astrophysique 
